\title{
Efectividad de la atención domiciliar de enfermería en el mejoramiento del autocuidado de personas adultas mayores ${ }^{1}$
}

Institución: Universidad de Costa Rica

\author{
Shirley Vargas Chaves ${ }^{2}$ \\ Jaime José Fernández Chaves ${ }^{3}$
}

\section{COMO CITAR}

Vargas, S. y Fernández, J.J. (2014). Efectividad de la atención domiciliar de enfermería en el mejoramiento del autocuidado de personas adultas mayores. Rev. Enfermería Actual en Costa Rica, 27, 1-11. DOI: http://dx.doi.org/10.15517/revenf.v0i27.16059

\section{RESUMEN}

Antecedentes. El objetivo de este artículo es presentar la mejor evidencia disponible en relación con el desarrollo de las visitas domiciliares de enfermería, brindadas a personas adultas mayores, con un enfoque de promoción de la salud y prevención de las enfermedades para el mejoramiento en su autocuidado. El proceso de envejecimiento poblacional en Costa Rica y a nivel mundial, como producto de los avances en condiciones socioeconómicas y sanitarias, ha generado necesidades para la atención de personas adultas mayores que permiten un mantenimiento o mejoramiento de la calidad de vida.

Método. como método se utilizó la investigación basada en la evidencia, la cual parte de una pregunta clínica; en cuanto a los datos, se realizó una búsqueda bibliográfica en las bases de datos EBSCO, PubMed y ProQuest, para luego ejecutar una lectura crítica con el programa FLC 2.0.

Resultados. Se obtuvo un total de 58 artículos, de cuya lectura crítica se seleccionó seis que presentaron la mejor evidencia.

Conclusión. Se concluye que las personas adultas mayores que reciben atención domiciliaria del profesional de enfermería desde un enfoque de la promoción de la salud y prevención de la enfermedad mejoran su autocuidado.

Palabras clave: atención-domiciliar-de-enfermería; autocuidado; personas-adultas-mayores; prevención-de-la-enfermedad; promoción-de-la-salud.

\footnotetext{
${ }^{1}$ Fecha de recepción: 30 de julio del 2014

Fecha de aceptación: 2 de setiembre del 2014

${ }^{2}$ Universidad de Costa Rica. Escuela de Enfermería. Costa Rica. Correo electrónico: shirley.vargaschaves@ucr.ac.cr

${ }^{3}$ Universidad de Costa Rica. Escuela de Enfermería. Costa Rica. Correo electrónico: jaimejose.fernandez@ucr.ac.cr
} 


\title{
Effectiveness of nursing home care in improving self-care of older persons ${ }^{1}$
}

\author{
Shirley Vargas Chaves ${ }^{2}$ \\ Jaime José Fernández Chaves ${ }^{3}$
}

Institution: Universty of Costa Rica

\section{CITED}

Vargas, S. y Fernández, J.J. (2014). Effectiveness of nursing home care in improving self-care of older persons. Rev. Enfermería Actual en Costa Rica, 27, 1-11. DOI: http://dx.doi.org/10.15517/revenf.v0i27.16059

\begin{abstract}
Background. The objective of this article is to present the best available evidence in relation to the development of nursing home visits, made to elderly, with a focus on health promotion and disease prevention for the improvement in self-care. The aging population in Costa Rica and worldwide, as a result of advances in socioeconomic and sanitary conditions, has led to the need for care of elderly individuals that allow maintaining or improving the quality of life.

Method. as a research was used method based on the evidence, which part of a clinical question; in terms of the data, a literature search in the databases EBSCO, ProQuest and PubMed data was performed, and then run a critical reading of the FLC 2.0.

Result. Was obtained a total of 58 items, whose critical reading was selected six presented the best evidence.

Conclusion. We concluded that older adults receiving home care nurse from an approach to health promotion and disease prevention improve self-care.
\end{abstract}

Key Words: disease; health promotion; home-care; older-people; self-care.

\footnotetext{
${ }^{1}$ Date of receipt: July 30, 2014

Date of acceptance: September 2, 2014

${ }^{2}$ Universidad de Costa Rica. Escuela de Enfermería. Costa Rica. Correo electrónico: shirley.vargaschaves@ucr.ac.cr

${ }^{3}$ Universidad de Costa Rica. Escuela de Enfermería. Costa Rica. Correo electrónico: jaimejose.fernandez@ucr.ac.cr
} 


\section{INTRODUCCIÓN}

El aumento de la población adulta mayor, tanto en el país como en el mundo, es reflejo de las condiciones sociales y de salud, entre las cuales puede identificarse una disminución en la tasa de fecundidad, así como un aumento en la esperanza de vida al nacer, principalmente en países desarrollados, los cuales comparten ciertas características con Costa Rica.

Sin embargo, dicha situación determina la necesidad de implementar estrategias para la promoción de la salud y prevención de las enfermedades en ese grupo, lo cual permite la transición de la enfermería tradicional asistencialista a una enfermería inmersa en un paradigma transformador, en el que la base de atención primaria de la salud permite fundamentar acciones en la atención de primer nivel, como las citadas.

La Organización Panamericana de la Salud/Organización Mundial de la Salud (s.f.) en La Renovación de la Atención Primaria de la Salud en las Américas (2007) define la atención primaria de salud como "la asistencia sanitaria esencial accesible a todos los individuos [todas las personas] y familias de la comunidad a través de medios aceptables para ellos, con su plena participación y a un costo asequible para la comunidad y el país" (p.4), lo cual confirma que la salud es un derecho fundamental de toda persona.

Por su parte, el Programa de Atención a las personas mayores en Atención Primaria de las Islas Canarias, España (2002), menciona que,

las características propias de atención primaria, en cuanto a accesibilidad geográfica y horaria, facilita el contacto entre las/os profesionales en salud y personas a lo largo de su ciclo vital. Esto, junto con el enfoque integral de la atención que se oferta, permite realizar actividades de promoción de la salud, de prevención de la enfermedad, educación sanitaria, asistenciales y reinserción en la comunidad de las personas adultas mayores. (p.8)

De acuerdo con lo anterior, la atención domiciliar de enfermería, en este caso, representa el primer contacto entre las personas en las comunidades con el sistema de salud, contacto en el que enfermería desempeña un papel sumamente valioso en la intervención con todos los grupos de la sociedad, integrada, entre otros grupos, por la población adulta mayor. Además, esta estrategia de atención de primer nivel de atención en salud, es un escenario de práctica clínica que amerita su estudio en varios ámbitos, tales como el impacto a nivel de autocuidado, que se refleja en la calidad de vida, según la Teoría General de Enfermería de Dorothea Orem.

Por otro lado, dicha estrategia se desarrolla tradicionalmente desde un enfoque asistencialista, en el que la intervención se enfoca en personas con condiciones de morbilidad, el cual ha sido un avance muy importante respecto de la atención de enfermería, la cual, sin embargo, también se desarrolla desde un punto de vista de la promoción de la salud y la prevención de la enfermedad, abordajes que corresponden a la atención de primer orden, en el que enfermería, con su fundamentación en la teoría mencionada, representa una ciencia y disciplina idónea para el desarrollo de estas estrategias. 
El objetivo del presente estudio consistió en identificar si el desarrollo de visitas domiciliares de enfermería para personas adultas mayores, con un enfoque de promoción de la salud y prevención de las enfermedades, permite un mejoramiento en su autocuidado.

\section{MATERIALES Y MÉTODOS}

Para efectuar la presente investigación, se aplicó la metodología de práctica clínica basada en la evidencia: la Enfermería Basada en la Evidencia (EBE) se refiere a la utilización consciente, explícita y juiciosa de la mejor evidencia científica disponible al tomar decisiones sobre el cuidado de los pacientes [de las personas] (Sackett, Richardson, Gray y Haynes, 1996, p.71), procedente de una investigación válida y fiable. Establece cinco pasos para su correcta aplicación:

1. Formulación de una pregunta de primera línea según el acrónimo PICO.

2. Búsqueda de información científica disponible.

3. Realización de un análisis crítico de los datos (lectura crítica).

4. Implementación de los resultados encontrados en el quehacer diario del profesional.

5. Evaluación de la implementación. manera:

La pregunta formulada fue creada según el acrónimo PICO, cuyo significado se desglosa de la siguiente

- P: Tipo de paciente o la patología de la que surge la pregunta. En este caso, personas adultas mayores.

- I: La intervención que se quiere analizar. Para el presente estudio, se refiere a las visitas de atención domiciliar de enfermería con un enfoque de promoción de la salud y prevención de la enfermedad.

- C: La comparación con otra intervención. Personas adultas mayores que reciben atención domiciliar de enfermería con un enfoque asistencial.

- O: Los resultados clínicos esperados. Mejoramiento del autocuidado.

El tipo de pregunta correspondió a una pregunta de terapia: ¿Las personas adultas mayores que reciben atención domiciliaria del profesional de enfermería desde un enfoque de la promoción de la salud y prevención de la enfermedad, respecto de la atención asistencial de enfermería, mejoran su autocuidado?

La pregunta planteada con el formato anterior funge como una guía clara para las personas que realizan el estudio, la cual, según Rojas, Cubero y Rodríguez (2013), asegura una buena búsqueda de información y por ende la mejor respuesta.

La fase posterior al planteamiento de la pregunta clínica corresponde a la búsqueda de la información científica disponible, en la que se utilizó descriptores en tres bases de datos del Sistema de Bibliotecas, Documentación e Información (SIBDI) de la Universidad de Costa Rica, las cuales fueron EBSCO-host, PubMed y ProQuest, que se especifican a continuación:

En ProQuest: "older people" AND "nursing home care" AND "health promotion" AND "disease prevention" AND "self-care". 


\section{.}

。

\section{Revista Electrónica Eneremeria Actual en costa Rica}

- $\quad$ Para la revisión realizada en EBSCO, se utilizaron los descriptores "older people" AND "nursing home care"

- Finalmente, los utilizados para la revisión en PubMed fueron los siguientes: elderly people, home care, nurse, health promotion, self care.

De los artículos encontrados (EBSCO: 31, PUBMED: 17 y PROQUEST: 10), la selección se llevó a cabo de acuerdo con el contenido y calidad de la evidencia científica, mediante el uso del programa FLC 2.0, procedimiento que se describe en la siguiente etapa del estudio. Para dicha sección, correspondiente al análisis crítico de la calidad de la información, se aplicó el programa FCL 2.0 versión 1.1.0, acrónimo de fichas de lectura crítica, el cual ha sido diseñado para,

facilitar el proceso de Lectura Crítica de literatura científica mediante la valoración de los elementos metodológicos básicos, así como agilizar el proceso de síntesis de la información mediante la generación automática de 'tablas de evidencia', en el contexto de revisiones sistemáticas de la evidencia (Osteba, 2008, p. 2).

Las tablas de evidencia a partir de los datos introducidos fueron diseñadas para facilitar los procesos de lectura crítica y de síntesis de la evidencia, y para valorar diferentes diseños de estudios. Dentro del proceso de revisión sistemática, la lectura crítica de la evidencia facilita el análisis de la calidad o validez de las pruebas científicas. Al respecto, cabe mencionar que se entiende por calidad de la evidencia o calidad de los estudios científicos, la confianza en que los sesgos potenciales del desarrollo del estudio hayan sido señalados de forma adecuada y en que los resultados y las recomendaciones sean válidos tanto interna como externamente. El proceso de lectura crítica implica no solo la valoración de los aspectos metodológicos más importantes, sino un análisis pormenorizado de los aspectos que contribuyen a la validez de un estudio.

En la etapa cuatro, correspondiente a la implementación, los resultados se socializan entre un grupo de profesionales de enfermería de diferentes instituciones a nivel nacional, con el propósito de mejorar las estrategias que hasta el momento se llevan a cabo, con el fin de favorecer la toma de decisiones oportuna y eficaz a partir de la mejor evidencia en atención domiciliaria para personas adultas mayores. Para concluir la etapa de la evaluación, en la cual se evalúa las estrategias que se van a implementar para identificar los cambios y si éstos han sido o no efectivos, se centrará en los resultados más que en el proceso.

Finalmente, con la mejor información se respondió a la pregunta planteada.

\section{Consideraciones éticas}

Se toma en cuenta que cada artículo que se utilizó en el desarrollo de la presente investigación cumpla con una estructura ética en cuanto al manejo de las personas participantes, así como con el uso de la información. A la vez se respetaron los derechos de autor, razón por la que se incorporó las citas bibliográficas correspondientes a lo largo del texto. 


\section{RESULTADOS}

De 31 estudios identificados en EBSCO con los descriptores mencionados, 29 fueron excluidos; mientras que de los 17 estudios identificados en PUBMED, 15 fueron excluidos y de los 10 estudios identificados en PROQUEST, 7 fueron excluidos; que no se considerara todos los artículos se debe a que no respondían a la pregunta PICO de forma directa ni indirectamente, o bien, por factores metodológicos de baja evidencia o no clasificables, que los convirtieron en inutilizables para la investigación.

\section{Se encontró la siguiente evidencia:}

1.Intervención en promoción de la salud para personas adultas mayores exitosas en tiempo corto, a partir de un estudio de tres partes en personas adultas mayores que viven en una zona de riesgo (Gustafsson, Wilhelmson, Eklund, Gosman, Zidén, Kronlof y Dahlen, 2012). Corresponde a un estudio aleatorio, ensayo de tres brazos, simple ciego y controlado, que se desarrolló entre noviembre de 2007 y mayo de 2011. Marco: los dos distritos urbanos de Gotemburgo, Suecia. Participantes: Cuatrocientos cincuenta y nueve adultos, residentes de la comunidad, con edades entre 80 y más años, no dependientes del servicio ayuda domiciliaria municipal. Intervención: Una visita domiciliaria preventiva o de cuatro reuniones de grupos de alto nivel, multiprofesionales, semanales, con una visita domiciliaria de seguimiento. Dicho estudio fue diseñado con el objetivo de examinar los resultados de las personas adultas mayores de la zona de riesgo, para evaluar si es posible retrasar el deterioro de las personas si una intervención de promoción de la salud se produce cuando una persona adulta mayor $(\geq 80)$ se encuentra en riesgo de que su salud se deteriore y si un grupo de intervención multiprofesional es más eficaz para retrasar el deterioro con una sola visita preventiva de origen con respecto a la fragilidad, además para indagar acerca de la autopercepción de la salud y las actividades de la vida diaria (AVD) a los 3 meses de seguimiento. Se concluye que las intervenciones promotoras de la salud, cuando las personas de este grupo de edad corren el riesgo de que su salud se complique, puede retrasar el deterioro de las actividades de la vida diaria; luego, en cuanto a la percepción de la salud, y a corto plazo, una intervención de grupo multiprofesional, como las reuniones de alto nivel descritas parece tener un mayor efecto en retrasar el deterioro en las ADL, en comparación con una sola visita domiciliaria preventiva. Se necesita más investigación para examinar los resultados a largo plazo y en diferentes contextos.

2. Una revisión de estudios de integración de las visitas domiciliarias preventivas entre las personas mayores - ¿es una perspectiva individual de los recursos de la salud, una visión o una realidad? (Fagerström, Wikblad y Nilsson, 2009). Esta investigación consiste en una búsqueda sistemática de la literatura en las bases de datos EBSCO, CINAHL, Medline, Science Direct y CSA, durante el periodo 1984-2004. El resultado de la búsqueda de la base de datos sistemática fue de un total de 49 artículos de investigación científica, de los cuales 18 están incluidos en este estudio, cuyos objetivos fueron: explorar y evaluar el efecto en la promoción de la salud de la visita domiciliaria preventiva (PHV) mediante el análisis de las actividades realizadas durante el PHV y los resultados reportados, y analizar críticamente si el PHV se caracteriza por una perspectiva individualizada de los recursos de salud. Se concluyó que el énfasis en enfermedad era sorprendentemente claro, y en 10 de 18 estudios faltaba un punto de vista de los recursos de salud, por tanto, los efectos de la PHV han sido cuestionados. A pesar de ello, los resultados actuales de la investigación implican que este método tiene un efecto positivo en la salud de las personas adultas mayores 
y su bienestar. Sin embargo, se subraya que la forma de atención aún debe ser desarrollada para incluir extensos exámenes e intervenciones, así como una perspectiva de recursos para la salud, en la que el punto de partida en cada visita domiciliaria son las necesidades y deseos individuales de la persona adulta mayor.

3. Promoción de la salud a nivel domiciliar para las personas mayores con enfermedades crónicas: resultados de un ensayo controlado aleatorio de un enfoque crítico de la reflexión (McWilliam et al., 1999). Los investigadores plantearon la hipótesis de que las personas adultas mayores con enfermedades crónicas que fueron intervenidas tendrían una actitud mucho más positiva (o actitud por la vida, la salud propia, y el cuidado de la salud), utilizarían mucho menos hospitales y servicios de atención domiciliaria, y tendrían mucha mejor calidad de la vida. El propósito de este estudio fue probar una intervención de promoción de la salud para las personas mayores de 65 años, dadas de alta del hospital, cuidadas en el hogar y con condiciones médicas crónicas. Se concluyó que los que recibieron la intervención tuvieron significativamente una mayor independencia y capacidad para gestionar su propia salud y mucho menos deseo de información inmediatamente después de la intervención. Tras un año de seguimiento, el patrón persistió, a pesar de las limitadas diferencias significativas referentes a la independencia y el deseo de información. Además, al año, el grupo de intervención redujo aún más la agencia de autocuidado y el locus de autoridad. La comparación de los cambios en el uso de recursos sanitarios por parte de los dos grupos no fue significativamente diferente. Sin embargo, el grupo de intervención tuvo una probabilidad significativamente mayor en cuanto a una mejor calidad de vida inmediatamente después de la intervención; la tendencia se mantuvo por un año, aunque las diferencias no fueron significativas. Los resultados indican el potencial clínico de reflexión crítica para mejorar la salud de las personas adultas mayores con enfermedades crónicas. Se necesita más investigación con las poblaciones menos frágiles.

4. Efectos de la práctica de enfermería avanzada en el hogar de la Salud, Programa de Consulta de las personas residentes en la comunidad mayores de 80 años y más (Imhof, Naef, Wallhagen, Schwarz y Mahrer, 2012). Se trata de un ensayo clínico aleatorizado, cuyo objetivo fue evaluar los efectos de una enfermera de práctica avanzada (APN) y del programa de consulta de salud en el hogar (HCP) en la calidad de vida, los indicadores de salud (caídas, eventos agudos), y la atención sanitaria de utilización. Se concluyó que tanto el Programa de Consulta de Salud en el hogar por parte de la enfermera de práctica avanzada, el cual implementa los principios de promoción de la salud, así como el empoderamiento, la asociación y la familia, pueden ser eficaces en cuanto a reducir los resultados adversos para la salud, tales como caídas, eventos agudos y hospitalizaciones.

5. Promoción de la salud en atención domiciliar para personas adultas mayores (Health promotion for frail older home care clients) (Markle, Weir, Browne, Roberts, Gafni y Henderson, 2006). Este ensayo controlado aleatorizado tiene como objetivo evaluar de manera comparativa los efectos y costos de la intervención proactiva de enfermería en promoción de la salud sumada a la intervención a la atención domiciliar usual para personas adultas mayores, con los servicios usuales de atención domiciliar. En el estudio se identificó que este tipo de intervenciones mejoran la calidad de vida de las personas que las reciben, además subraya la importancia del estudio en promoción de la salud en visitas domiciliares de Enfermería. 
6.Estudio randomizado sobre la prevención de discapacidad en personas adultas mayores habitantes de comunidad, con bajo y alto riesgo de institucionalización en centros donde se atiende a personas adultas mayores. Este estudio randomizado estratificado tuvo como objetivo analizar el impacto de los resultados de dos diferentes procesos de intervención de enfermería en personas adultas mayores residentes en Bern, Suiza. Los datos sugieren que esta intervención puede reducir las discapacidades en personas adultas mayores con bajo riesgo, pero no aquellas con un alto riesgo de deterioro funcional; dicho efecto está relacionado con las habilidades y destrezas de la persona que desarrolle la intervención domiciliar de enfermería.

\section{DISCUSIÓN}

La atención domiciliaria del profesional de enfermería desde un enfoque de la promoción de la salud y prevención de la enfermedad dirigida a las personas adultas mayores ayuda a la persona adulta mayor y a su familia a alcanzar un mayor nivel de independencia, ideal para potenciar el autocuidado.

Al respecto, es fundamental considerar que la atención domiciliaria ha experimentado cambios significativos a raíz de la adopción del nuevo modelo de atención, originado de la declaración de Alma Ata en OPS(1978), a partir de la que se pasó de un modelo basado en la asistencia médica primaria a uno de atención primaria de salud. En este mismo sentido la atención domiciliaria, tal como se entiende actualmente, es el fruto de pasar de una asistencia domiciliaria muy medicalizada e individualizada a una atención domiciliaria más holística y dirigida tanto al individuo [a la persona] como a la familia, entre otras diferencias (Martínez y Del Pino, 2012).

Sin embargo, según los autores anteriormente citados, en el presente la atención domiciliaria desde un enfoque de la promoción de la salud y prevención de la enfermedad, ha sido una actividad residual o secundaria por parte de los equipos de atención primaria en general y de profesionales en enfermería comunitarios en particular.

En Costa Rica, los servicios básicos de salud que realizan acciones de promoción de la salud, prevención de la enfermedad, curación y rehabilitación de menor complejidad son denominados establecimientos de salud del primer nivel de atención. Para efectos de la Caja Costarricense de Seguro Social (CCSS), el territorio nacional se divide en 7 regiones de salud, las cuales se subdividen en áreas de salud cuya población oscila entre 15 mil y 40 mil habitantes en las zonas rurales y entre 30 mil y 60 mil habitantes en las zonas urbanas; a cada una se le asigna un profesional en enfermería para que colabore con acciones de supervisión técnica y administrativa, educación continua y atención de referencia de casos por parte de los E.B.A.I.S. A su vez, cada una de las áreas de salud se subdivide en dos o más sectores de salud que son divisiones geográficas en las que, en promedio, viven entre 4000 y 4500 personas; cada sector de salud está a cargo de un equipo de recursos humanos denominado Equipo Básico de Atención Integral en Salud (E.B.A.I.S.), integrado, al menos, por 1 médico (a) general, 1 auxiliar de enfermería, 1 asistente técnico de atención primaria en salud (A.T.A.P.S.). Los ATAPS se encargan de la actividad central de la atención primaria, la visita a domicilio, de lo cual se colige que en Costa Rica la atención domiciliaria no es dada por profesionales de enfermería a nivel del sistema nacional de salud pública (García, 2004, p.p. 34-35). 
Sin embargo, los cambios demográficos, sociales y económicos conducen a una reflexión respecto de cuán urgente es un cambio sustancial en el posicionamiento, así como en la actitud ante la atención domiciliaria costarricense y de la importancia de esta en relación con los diferentes sectores del sistema de salud (Martínez y Del Pino, 2012).

Los diferentes estudios incluidos en esta revisión sistemática son suficientes para afirmar que las personas adultas mayores que reciben atención domiciliaria del profesional de enfermería desde un enfoque de la promoción de la salud y prevención de la enfermedad, sí mejoran su autocuidado, respecto de la atención meramente asistencial de enfermería.

En relación con lo mencionado, se indica que las personas que reciben una intervención basada en un enfoque crítico de la reflexión tienen una mayor independencia y capacidad percibida para gestionar su propia salud y mucho menos deseo de información inmediatamente después de tal proceso; por ende, afirman que este método tiene un efecto positivo en la salud y bienestar de las personas adultas mayores. Sin embargo, subrayan que la forma de atención aún debe ser desarrollada para incluir exámenes e intervenciones más extensas de las que se realizan actualmente, así como la inclusión de la perspectiva de recursos para la salud, pero partiendo que en cada visita domiciliaria son las necesidades, y deseos individuales de la persona adulta mayor, las que deben prevalecer. Además, añaden que se necesita más investigación con las poblaciones menos frágiles.

También propicia la estrategia a través de un Programa de Consulta de Salud en el hogar por parte de la enfermera de práctica avanzada, en la que se transmite los principios de promoción de la salud, el empoderamiento, la asociación y la familia-centrado, eficaces para disminuir los resultados adversos para la salud, tales como caídas, eventos agudos y las hospitalizaciones.

\section{CONCLUSIONES}

Las personas adultas mayores que reciben atención domiciliaria del profesional de enfermería desde un enfoque de la promoción de la salud y prevención de la enfermedad, mejoran su autocuidado.

La atención domiciliar por parte de la persona profesional en enfermería de práctica avanzada, guiada por los principios de promoción de la salud, el empoderamiento, la asociación y centrada en la familia, puede ser eficaz en la reducción de los resultados adversos para la salud, tales como caídas, eventos agudos y las hospitalizaciones. fragilidad.

Se requiere de más investigación respecto de las personas adultas mayores que presentan menor grado de

\section{REFERENCIAS BIBLIOGRÁFICAS}

Carol, L., McWilliam, C., Stewart. M., Belle, J., McNair, S., Donner, A., Desai, K., Coderre, P., Galajda, J. (1999).Home-based health promotion for chronically ill older persons: results of a randomized controlled trial of a critical reflection approach Health Promot. Int. 14 (1): 2741 doi:10.1093/heapro/14.1.27 
Fagerstrom, L., Wikblad, A., Nilsson, J. (2009). An integrative research review of preventive home visits among older people--is an individual health resource perspective a vision or a reality? Scandinavian Journal of Caring Sciences; 23(3):558-68. doi: 10.1111/j.1471-6712.2008.00637.x

García, R. (2004). Módulo Uno: El Sistema Nacional de Salud en Costa Rica: Generalidades. San José: Editorial Centro de Desarrollo Estratégico e Información en Salud y Seguridad Social (CENDEISSS).

Gustafsson, S., Wilhelmson, K., Eklund, K., Gosman, G., Zidén, L., Kronlöf, G. H., Dahlin, S. (2012). HealthPromoting Interventions for Persons Aged 80 and Older Are Successful in the Short Term-Results from the Randomized and Three-Armed Elderly Persons in the Risk Zone Study. Journal of the American Geriatrics Society, 60(3), 447-454. doi:10.1111/j.1532-5415.2011.03861.x

Imhof, L., Naef, R., Wallhagen, M., Schwarz, J., Mahrer R.(2012). Effects of an advanced practice nurse in-home health consultation program for community-dwelling persons aged 80 and older. J Am Geriatr Soc. 2012 Dec; 60 (12):2223-31.

Markle, M., Weir, R., Browne, G., Roberts, J., Gafni, A., y Henderson, S. (2006). Health promotion for frail older home care clients. Journal of Advanced Nursing, 54(3), 381-395. doi:10.1111/j.1365-2648.2006.03817.x

McWilliam, C., Stewart, M., Brown, J., McNair, S., donner, A., Desai, K., Coderre, P., \& Galajda, J.(1999). Home-based health promotion for chronically ill older persons: results of a randomized controlled trial of a critical reflection approach. HEALTH PROMOTION INTERNATIONAL. Oxford University Press, 14(1), 27-41

Martínez, J., Del Pino, R. (2012). Enfermería en atención primaria. Barcelona: Difusión Avances de Enfermería.

Organización Panamericana de la Salud/Organización Mundial de la Salud (OPS/OMS). (2007). Renovación de la atención primaria de salud en las Américas. Recuperado de: http://cursos.campusvirtualsp.org/file.../md3-lprenovacion-APS-spa-2007.pdf

Organización Panamericana de la Salud. (1978). Conferencia Internacional sobre Atención Primaria de Salud, Alma-Ata, URSS, 6-12 de septiembre de 1978.

Osteba (2008). FLC Critica Manual de Uso: Versión 1.1.0. Servicio de Evaluación de Tecnologías Sanitarias. Gobierno Vasco.

Rojas, V., Cubero, C., Rodríguez, A. (2013). La investigación en enfermería desde la perspectiva de la evidencia científica: un camino para el mejoramiento de la práctica clínica segura. San José: CIEBE-CR.

Sackett, D.., Rosenberg, W., Gray, J., Haynes, R., Richardson W. (1996). Evidence based medicine: what it is and what it isn't. BMJ. Jan 13; 312(7023): 71-2 


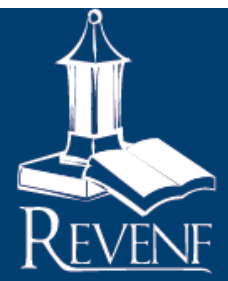

Servicio Canario de Salud (2002). Programa de Atención a las personas mayores en Atención Primaria. Servicio Canario de Salud, Consejería de Sanidad y Consumo del Gobierno de Canarias. Recuperado de: http://www2.gobiernodecanarias.org/sanidad/scs/content/9e6993c5-ec54-11dd-9b8199f3df21ba27/PROGRAMA_PERSONAS_MAYORES.pdf

Stuck, A., Minder, C., Peter, I., et al. A Randomized Trial of In-Home Visits for Disability Prevention in Community-Dwelling Older People at Low and High Risk for Nursing Home Admission. Arch Intern Med. 2000; 160 (7):977-986. doi:10.1001/archinte.160.7.977 